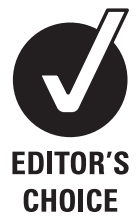

${ }^{1}$ University of California, Irvine, USA

2University of Southern California, Los Angeles, California, USA

Correspondence to María M Corrada 1513 Hewitt Hall, Irvine, CA 92697-1400, USA; mcorrada@uci.edu

Cohort study

\title{
Being overweight in adults aged $70-75$ is associated with a reduction in mortality risk compared with normal BMI
}

\section{María M Corrada, ${ }^{1}$ Annlia Paganini-Hill ${ }^{2}$}

\author{
Commentary on: Flicker L, McCaul KA, Hankey GJ, et al. Body mass index and survival in men and \\ women aged 70 to 75. J Am Geriatr Soc 2010;58:234-41.
}

The prevalence of overweight and obesity is increasing across the whole age range, including among older people, in both developed and developing countries. Increased weight, especially obesity, is a wellestablished risk factor for cardiovascular disease and all-cause mortality in adults. However, the current standard categories of overweight and obesity based on body mass index (BMI), which were established to reflect such increased morbidity and mortality, are based largely on studies in young and middle-aged adults. As shown by the accompanying study and previous reports, these categories may not be appropriate for older people.

\section{The study by Flicker and colleagues}

Flicker and colleagues examined the association between BMI and mortality in a sample of older people aged 70-75 years. The participants were 4677 men and 4653 women living in metropolitan and urban areas of Australia from two different population-based longitudinal studies of health, The Health in Men Study and the Australian Longitudinal Study of Women's Health. BMI was calculated from self-reported measures of height and weight and demographic, lifestyle and health status characteristics were ascertained. The participants were followed for 10 years and specific causes of death were recorded. The main objective of the study was to determine the BMI level associated with the lowest mortality. Mortality was in fact lowest for overweight participants in whom the risk of death was about 13\% less than that of normal weight participants. The result was consistent in men and women, the healthy and unhealthy, sedentary and non-sedentary persons and for all causes of death studied. The authors concluded that the BMI thresholds for overweight and obese are too restrictive for older people.
The study has some limitations including a relatively narrow age range, the low response rate, especially for women, and the lack of information on race or ethnicity, putting in question the generalisibility of the results to the general older population. Additional limitations in this and other similar studies include the estimation of BMI from self-reported height and weight, the lack of information on weight fluctuation or intentional versus unintentional weight loss and the use of BMI as a measure of body fat. In contrast, the study has many strengths including its large population-based sample with complete follow-up and the detailed analytic approach. The authors used sensitivity analyses to determine the best shape for the association between BMI and mortality, performed analyses to minimise the influence of pre-existing illness and early mortality and examined a variety of potential confounders and effect modifiers.

\section{Current BMI overweight standard not appropriate for older people}

The results from this well-executed study add to the ever-growing body of evidence suggesting that current weight standards may not be appropriate for older people. Many studies of BMI and mortality in older people in North America, Europe, Australia, New Zealand and Asia had similar results. A systematic review ${ }^{1}$ of 13 studies in people aged 65 and older was undertaken specifically to challenge the appropriateness of current weight guidelines for older people. A meta-analysis ${ }^{2}$ of 28 studies updated the systematic review and addressed some of its limitations. The overall conclusion was that in older men and women a BMI in the overweight range is not associated with increased mortality and that obesity is associated with only a modest (about 10\%) increase in mortality. 
Although the current BMI thresholds have been recommended for all individuals aged 18 or older, this one-size-fits-all category may not be appropriate for older people.

\section{Is it time for new age-specific guidelines?}

Although many agree that BMI is not an ideal measurement of body fat and it is debated whether its seemingly paradoxical association with mortality in older people is real or due to a variety of biases, ${ }^{3}$ the reality is that it is easy, inexpensive and in widespread use. Studies suggest that among older people, weight loss may be related to increased mortality regardless of initial weight. ${ }^{4}$ So as long as physicians use BMI to give older people advice regarding weight loss, it is important this advice is based on evidence relevant to this age. It is time for health policy decision makers to look at the large body of evidence now available to decide whether changes in ideal weight recommendations are warranted for older people.

\section{Competing interests None.}

\section{References}

1. Heiat A, Vaccarino V, Krumholz HM. An evidence-based assessment of federal guidelines for overweight and obesity as they apply to elderly persons. Arch Intern Med 2001;161: 1194-203.

2. Janssen I, Mark AE. Elevated body mass index and mortality risk in the elderly. Obes Rev 2007;8:41-59.

3. Oreopoulos A, Kalantar-Zadeh K, Sharma AM, et al. The obesity paradox in the elderly: potential mechanisms and clinical implications. Clin Geriatr Med 2009;25: 643-59, viii.

4. Newman AB, Yanez D, Harris T, et al. Weight change in old age and its association with mortality. $J$ Am Geriatr Soc 2001;49:1309-18. 